Books, videos, CD-ROMs, DVDs and any other relevant items submitted for a review in the $B D J$ should be addressed to: Kate Maynard, Assistant Editor, British Dental Journal, NPG, 4-6 Crinan Street, London N1 9XW

\section{Textbook of oral and maxillofacial anatomy, histology, and embryology}

\author{
S. R. Pabhu \\ UK: Oxford University Press \\ price $€ 15.99$, pp 286
}

ISBN 0195665112

This is the third in a series of concise dental textbooks with the other two devoted to contemporary dental materials and oral medicine. The editor is Professor of Oral Medicine at the University of the West Indies with a depth of clinical experience and of teaching in a number of diverse environments. He has brought together 12 other contributors of varying seniority from India, Australia, Kuwait, Malaysia and the USA. The stated aim of the book is to cover topics that are taught and examined during the first and second years of the dental course being based on curricular guidelines of a number of dental councils and universities.

The 45 relatively short chapters are grouped under the three main headings of maxillofacial and oral anatomy, histology and embryology. Anatomy is considered in two sections covering the head and neck and oral cavity and dentition with embryology also subdivided into maxillofacial and oral and the teeth and supporting structures.

The text is written in a clear and concise manner with suggestions for further reading spread throughout. A valuable feature is the inclusion of short paragraphs on clinical implications at the conclusion of a number of chapters. The book is well illustrated and although the line drawings and photomicrographs within are in monochrome while those on the cover are in colour, they are clearly laid out and generally well labelled or alternatively accompanied by detailed captions. The overall standard of presentation is generally very good and the index well set out. For future editions however it may be helpful to address the occasional typographical error and also in the description of lymphatic drainage of the head and neck to mention the important clinical levels I-V.

Although primarily targeted at undergraduate students of dentistry and also those studying dental technology, dental hygiene and nursing, there is much material that would be of considerable value for candidates preparing for postgraduate examinations such as the MFDS and MFGDP. In my opinion the stated aims are certainly met as a studentfriendly and ex-orientated volume which I would highly recommend.

J. Lowry

\section{Clinical success in early orthodontic treatment}

\author{
A. Patti, G. Perrier D'Arc \\ UK: Quintessence \\ price $£ 48.00$, pp 124 \\ ISBN 2912550378
}

This colourfully illustrated text describes the circumstances when early interceptive orthodontics becomes appropriate. It explains how the clinician may alter an unfavourable growth pattern in the young orthodontic patient by interceptive treatment in the periods of rapid growth, thus reducing the amount of subsequent orthodontic treatment. Provided the clinician is appreciative of the influencing factors determining various malocclusions, the patient may be managed appropriately because 'it is better to prevent than cure'.

The opening chapter briefly summarises the morphogenesis of the facial skeleton. The authors highlight the importance of assessing the patient to determine the facial type ie brachyfacial, dolichofacial and mesofacial. Each individual is different so growth rate and rhythm must be determined for each patient to prescribe an appropriate treatment plan.

The purpose and function of the primary dentition are described in the second chapter. Predictors for possible future malocclusions are described and followed up with comprehensive diagrams, which is consistent throughout the text.

Interferences with the normal development of the dentofacial structures have consequences for the associated structures. Mechanical interferences operate in three different planes - transverse, saggital and vertical - and are described in the book eg anterior and buccal crossbites, increased overjet/ overbite and anterior open bites. The consequences of mouth breathing are discussed eg hypotonia of the nares, poor posture, sleep apnoea and a shortened upper lip. The maintenance of the infantile swallowing tongue-thrust may lead to anterior open bite and procliniation of the maxillary incisors.

An in depth approach to clinical examination is described. Study models, radiographs and clinical photographs all contribute to the diagnostic examination. A useful checklist for analysing lateral cephalometric radiographs is given at the end of the fourth chapter. There is an algorithm to aid clinicians with the diagnosis and treatment planning.

'Unlocking', the term used to describe removal of mechanical and functional interferences, and the principles of 'unlocking', give the clinician a guide for early treatment objectives. There is a logical progression through the malocclusions which affect Class I, II and III. Handy checklists for the indications and contra-indications for serial extractions are useful for the clinician who cannot resolve crowding in the mixed dentition as extracting a permanent tooth may be required. The anomalies of the primary dentition and the mode of treatment to resolve them are described. Early signs of primary crowding of the deciduous dentition are listed and the management from the removal of interproximal enamel of the primary molars to the use of the Crozat quadhelix in the mildly crowded. Secondary crowding eg skeletal and functional open bites, diastemas, increased overbites, 

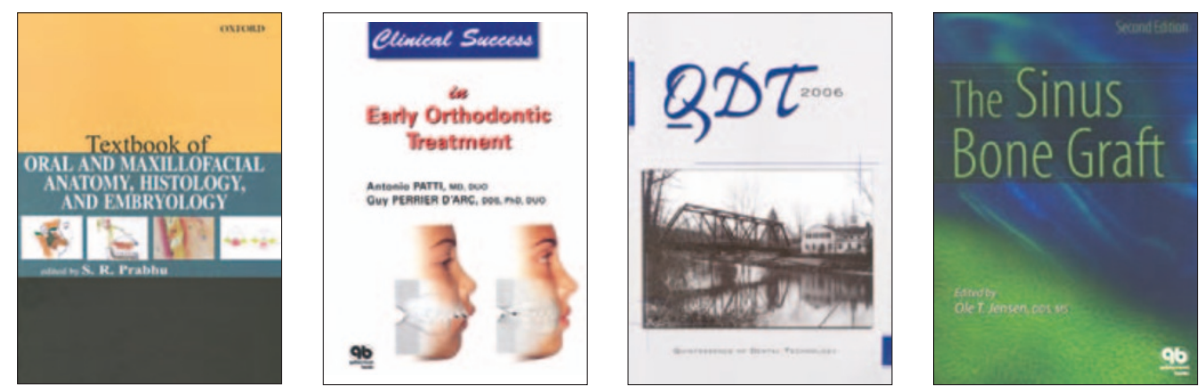

crossbites and transverse anomalies are described and their relevant treatment modalities to manage these malocclusions. Characterisitics of Class II and Class III malocclusions are discussed and the suitable management for each situation.

The information is beyond the depth of knowledge for undergraduate studies or MFDS examinations. However, this is an interesting textbook which would be appropriate for Orthodontic Specialist Registrars or postgraduate dentists with a special interest in interceptive orthodontics.

\section{E. Henley}

\section{Quintessence of dental technology 2006}

\author{
A. Sadan (Ed) \\ UK: Quintessence \\ price $\mathrm{E} 45.00$, pp 228 \\ ISBN 0867154594
}

Quintessence of Dental Technology is a journal which is published annually. It contains 20 articles on a wide range of subjects within prosthodontics and dental technology. As might be expected from Quintessence, it is beautifully produced with excellent photographic illustrations. Most of the articles are jointly authored by clinicians and technicians and are based on one or more case studies. In an era in which emphasis is placed on evidence-based research, this publication fills a gap in the literature. Its aim is to show advanced dental technology at its best. The topics covered range from ceramics through dental technician training to implantology, prosthodontic rehabilitation with surgical intervention and rehabilitation with immediate replacement dentures.

The clinical procedures described are mostly standard, only the laboratory techniques being new, usually as a result of the use of newer materials such as zirconium oxide ceramic. An article on cavity preparation for indirect partialcoverage restorations bases cavity design for ceramic inlays on anatomical and structural considerations. Thirty years ago treatment of the hopeless dentition with immediate dentures was routine and unlikely to command much space in the literature. Is it a measure of how far dentistry has come that alongside advanced ceramics and implantology, one article describes two such cases?

At US\$76 it is not easy to predict who might buy this, but it is to be hoped that dental school libraries might subscribe to enable dental students to see what is possible in restorative dentistry. It might also provided a timely reminder to dental deans and curriculum planners of the importance of dental technology and of the link between clinician and technician.

R. Clark

\section{The sinus bone graft (second edition)}

\section{T. Jensen \\ UK: Quintessence \\ price $€ 107.00$, pp 384 \\ ISBN 0867154551}

Oral implants are today a popular treatment modality for the prosthetic rehabilitation of an edentulous space. The placement of implants in the maxillary sinus region can be a daunting task for general dental practitioners and often results in a referral to secondary or specialist centres. This book focuses on this topic and aims to give the clinician both a scientific and clinical overview to help overcome this challenging scenario.

It is clear from the outset that the principal author has extensively researched the literature and sought the opinion of leading clinicians regarding sinus bone grafts. The book has numerous worldwide contributors whose clinical specialities include oral and maxillofacial surgery, periodontics, implantology and general practice. The contents of the book are divided into four clear logical sections which are further subdivided into chapters. The first section takes the clinician through the scientific background of sinus bone grafts. In particular there are very useful chapters describing the indications and contraindications of this technique which can aid case selection. The possible complications of sinus grafts are also detailed. This is of great value not only for assisting the clinician but more importantly contributing to the information provided to the patient during the consenting process. The second section concentrates on the various sinus bone grafting materials and sources. Autologous, allograft, alloplasts and xenografts are all described and the surgical harvesting and placement of these grafts are demonstrated very well. As the reader will soon realise, all information is well supported by clinical evidence and presented in an easy to understand fashion. The third section gives an overview of auxiliary procedures used for sinus augmentation. Science never stands still and section four gives the reader an insight into the future of bone grafting techniques. Topics such as gene therapy, use of stem cells and tissue engineering are discussed.

The cover and general outline of the book have a contemporary design which enhances its readability. The text in each section is clear and concise. The book contains a plethora of excellent clinical photographs and radiographs which are well supported by colour line illustrations.

Overall this textbook achieves its objectives and provides a comprehensive review. It has a broad appeal and will make a valuable addition to the library of general dental practitioners and specialist oral surgeons who have an interest in implantology.

J. Seehra 